\title{
When trichoscopy enlightens clinic: Fortuitous finding of tinea capitis on alopecia of lupus discoid
}

\section{Selma Benkirane, Mounia Bennani, Jihane Ziani, Zakia Douhi, Sara Elloudi, Hanane BayBay, Fatima Zahra Mernissi}

\author{
Department of Dermatology, University Hospital Hassan II, Morocco
}

Corresponding author: Dr. Selma Benkirane, E-mail: dr.benkiraneselma@gmail.com

Sir,

A 19-year-old patient, with no notable pathological antecedents, followed in our training for chronic discoid-type lupus retained on clinical, histological and immunological criteria using synthetic antimalarials, with clinical involvement of the isolated scalp, histological examination showed an epidermis regularly atrophic and surmounted by an ortho-keratotic keratosis, a lymphocytic inflammatory infiltrate in the dermis disposed around the hair follicles and sometimes perivascular and vacuolar alterations of the basal layer. During a routine hospitalization for systematization assessment, examination of the scalp showed several cicatricial and non-cicatricial alopecia plates, weakly limited, asymptomatic resting on a slightly erythematous background evolving for 1 year worsened for 2 months (Fig. 1). The rest of the clinical examination was unremarkable. The dermoscopic examination (Fig. 2), revealed, on a background of erythema congestive, large yellow-orange dots with white perifollicular halos, (Fig. 2: yellow circle) thick linear and sinuous arborescent vessels (Fig. 2: Red circle) snaking between the follicular orifices as well as commas (Fig. 2: Blue arrow), hair in arobase (Fig. 2: purple arrow), hair zigzag, and short broken hair (Fig. 2: green arrow]. In front of the trichoscopic aspect suspecting a tinea capitis, mycological sampling was performed (Fig. 3) income in favor of a trichophyton rubrum put under griseofulvin oral and topical as well as rules of adapted hygiene with a good clinical evolution (Fig. 4) and disappearance of trichoscopic signs (Fig. 5) after six weeks of treatment.

The involvement of the scalp is frequent and inaugural in more than half of cases of chronic lupus

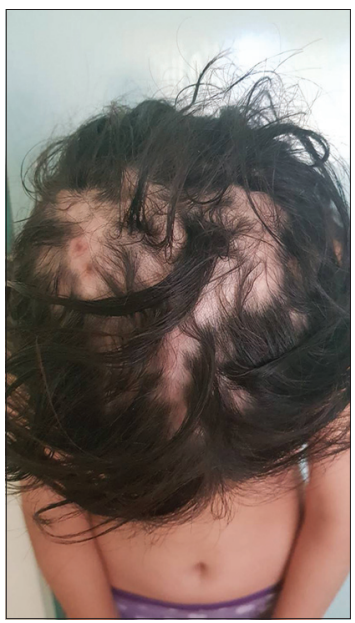

Figure 1: Clinical picture of cicatricial and non-cicatricial alopecia of the scalp.

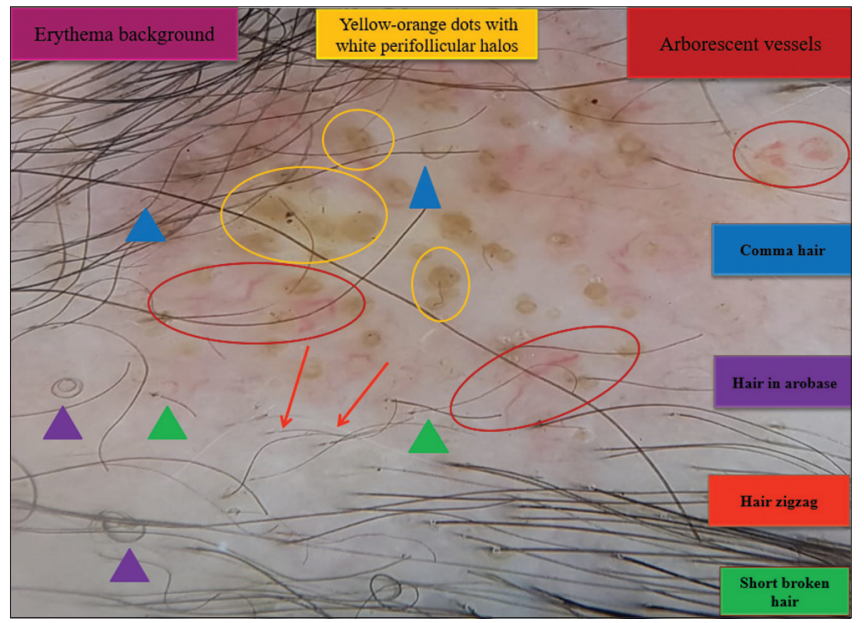

Figure 2: Trichoscopy associating signs in favor of tinea capitis and lupus.

erythematosus and can remain isolated, as in the case of our patient, in $10 \%$ of cases [1]. Tinea capitis, for its

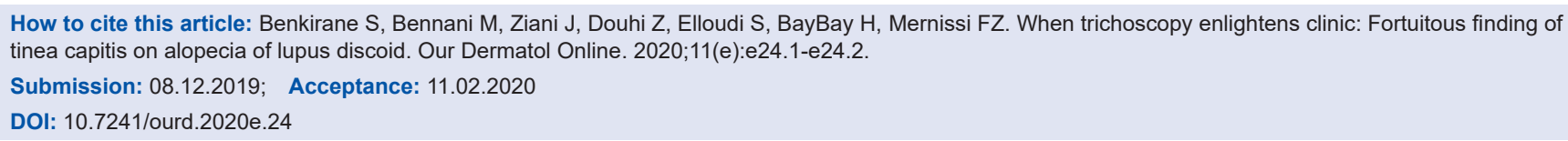




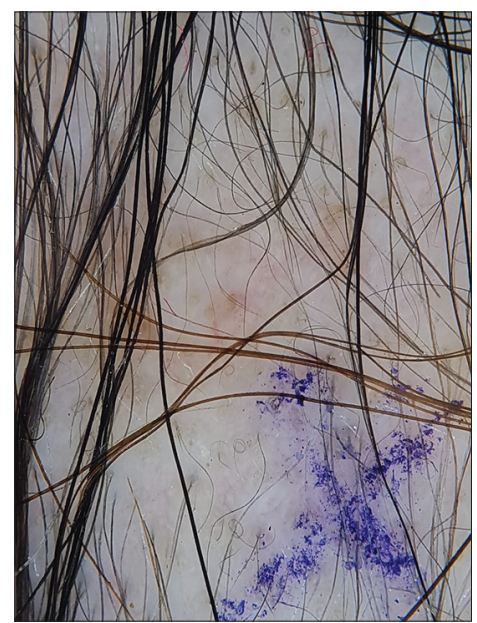

Figure 3: Mycological sampling directed by the dermoscope.

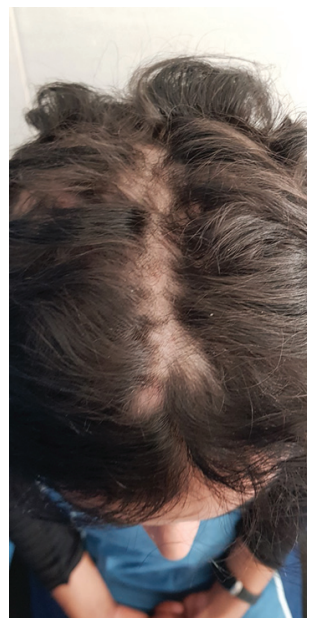

Figure 4: Clinical control after six weeks.

part, is a fungal infection that remains rare in adults and affects mainly women aged 19 to 38 years. The rarity of TC in adults is not well understood but can be explained by the fungistatic properties of long-chain fatty acids in post-pubertal sebum, the maturation of hair follicles and the immune system after age. adult that could protect against fungal invasion $[2,3]$. The clinical presentation is generally atypical in adults. Dermoscopy is a non-invasive tool that can help clinicians make the diagnosis. The most specific signs described were comma hair, corkscrew hair, zigzag hair and Morse code. The purpose of this presentation is to show the interest of trichoscopy for the diagnostic

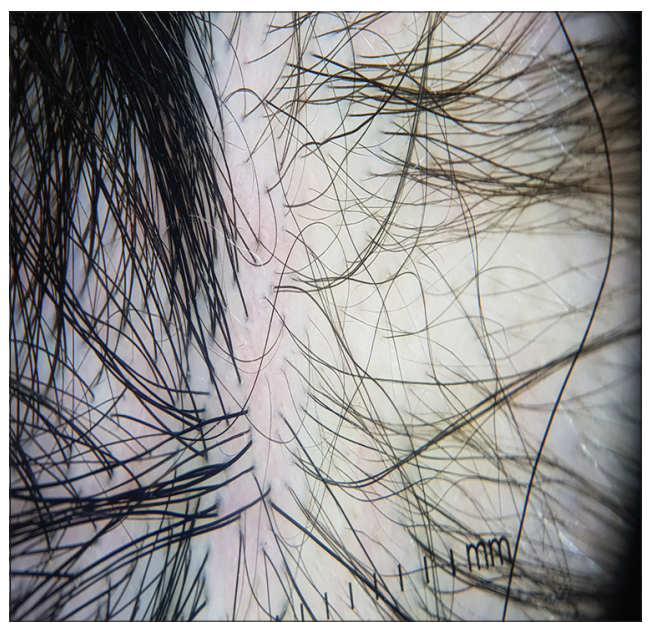

Figure 5: Disappearance of trichoscopic signs cited above after six weeks of treatment.

orientation of alopecia as well as for therapeutic evaluation.

\section{Consent}

The examination of the patient was conducted according to the Declaration of Helsinki principles.

The authors certify that they have obtained all appropriate patient consent forms. In the form the patient(s) has/have given his/her/their consent for his/her/their images and other clinical information to be reported in the journal. The patients understand that their names and initials will not be published and due efforts will be made to conceal their identity, but anonymity cannot be guaranteed.

\section{REFERENCES}

1. Francès C. Manifestations cutanées des lupus érythémateux. EMCDermatologie. 2015;10:1-14.

2. El-Khalawany M, Shaaban D, Hassan H, Abdalsalam F, Eassa B, Abdel Kader A, et al. A multicenter clinicomycological study evaluating the spectrum of adult tinea capitis in Egypt. Acta Dermatovenerol Alp Pannonica Adriat. 2013;22:77-82.

3. Cervetti O, Albini P, Arese V, Ibba F, Novarino M, Panzone M. Tinea capitis in adults. Adv Microbiol. 2014;4:12.

Copyright by Selma Benkirane, et al. This is an open access article distributed under the terms of the Creative Commons Attribution License, which permits unrestricted use, distribution, and reproduction in any medium, provided the original author and source are credited.

Source of Support: Nil, Conflict of Interest: None declared. 